\title{
The response to combination therapy treatment regimens in severe/difficult-to-
} treat asthma

\author{
J.D. Campbell*, L. Borish", T. Haselkorn`, L. Rasouliyan ${ }^{+}$, J.H. Lee`, S.E. Wenzel ${ }^{\S}$ \\ and S.D. Sullivan* on behalf of the TENOR Study Group
}

ABSTRACT: The aim of the present study was to assess the response of high-dose salmeterol/ fluticasone combination (SFC) and low-dose SFC compared with regimens without inhaled corticosteroid (ICS) plus long-acting $\beta$-agonist (LABA) in a large cohort with severe or difficult-totreat asthma.

Subjects were administered low-dose SFC (100/50 or 250/50 $\mu \mathrm{g})$ or high-dose SFC (500/50 $\mu \mathrm{g})$, and a control group received medications that could include ICS or LABA but not both. The present authors calculated unadjusted and propensity score-adjusted differences in outcomes consistent with components of asthma control, comparing high-dose and low-dose SFC cohorts with controls.

The low-dose SFC cohort had higher asthma-related quality of life and fewer asthma control problems compared with controls. The high-dose SFC cohort had higher forced expiratory volume in one second but higher odds of having severe asthma compared with controls.

The present results support the evidence that some asthmatics achieve better outcomes while receiving a low-dose salmeterol/fluticasone combination, but also suggest that those on a highdose salmeterol/fluticasone combination fail to achieve significant improvement in many controlrelated health outcomes as compared with similar patients not receiving salmeterol/fluticasone combination. These findings suggest a limited value of high-dose salmeterol/fluticasone combination compared with the alternatives. While additional studies are needed, the present findings call for alternative therapeutic approaches in severe/difficult-to-treat asthma for those unable to attain asthma control with or without salmeterol/fluticasone combination.

KEYWORDS: Asthma control, combination therapy, difficult-to-treat, salmeterol/fluticasone combination, severity

sthma treatment guidelines from the National Asthma Education and Prevention Program [1] and the Global Initiative for Asthma [2] and recommend inhaled corticosteroids (ICS) plus a long-acting $\beta$-agonist (LABA) as the preferred treatment regimen in patients with moderate or severe persistent asthma who are not adequately controlled on ICS therapy alone. These recommendations were based on several large pivotal randomised clinical trials that demonstrated, through the primary efficacy outcome of pulmonary function improvements, the superiority of adding LABA to ICS for the management of mild-to-moderate persistent asthma [1, 2]. Salmeterol/fluticasone combination (SFC), an aerosol containing a LABA (salmeterol) and an ICS (fluticasone), is indicated for the maintenance treatment of asthma in patients with reversible obstructive airway disease when the use of a combination product is considered to be appropriate [3]. Studies have demonstrated that low-to-intermediate doses of SFC (100/50 or $250 / 50 \mu$ b.i.d) confer greater clinical benefit for mild-to-moderate asthma when compared with increased doses of an ICS or the addition of a leukotriene modifier to an ICS $[3,4]$.

There is a lack of evidence that demonstrates the superiority of high-dose SFC (500/50 $\mu \mathrm{g}$ b.i.d) compared with its alternatives (such as high-dose ICS therapy or high-dose ICS plus other non-LABA long-term controller therapies) in adults with severe or difficult-to-treat asthma, patients who bear the greatest burden of the disease. In a randomised trial comparing high-dose SFC (500/50 $\mu \mathrm{g}$ b.i.d) to both
AFFILIATIONS

*University of Washington, Seattle, WA,

\#University of Virginia Health Systems, Charlottesville, VA,

"Genentech Inc., and

${ }^{+}$ICON Clinical Research, San

Francisco, CA, and

${ }^{\S}$ University of Pittsburgh, Pittsburgh, PA, USA.

CORRESPONDENCE

J.D. Campbell

Pharmaceutical Outcomes Research and Policy Program

University of Washington

1959 NE Pacific Ave

H375Q

Box 357630

Seattle

WA 98195

USA

Fax: 12065433835

E-mail: campbeli@u.washington.edu

Received:

August 272007

Accepted after revision:

July 202008

\section{STATEMENT OF INTEREST}

Statements of interests for all of the authors and the study itself can be found at www.erj.ersjournals.com/ misc/statements.shtm 


\begin{tabular}{|c|c|c|c|c|c|}
\hline Characteristics & Never $^{\#}$ & Low-dose & High-dose & Low & High \\
\hline Subjects n & 208 & 205 & 271 & & \\
\hline Age yrs & $51.0 \pm 114.49$ & $51.8 \pm 113.35$ & $49.2 \pm 113.35$ & 0.574 & 0.152 \\
\hline White & $180(86.5)$ & $174(84.9)$ & 209 (77.1) & 0.630 & 0.009 \\
\hline Smoking status & & & & 0.510 & 0.869 \\
\hline Never & $140(67.3)$ & 127 (62.0) & 177 (65.3) & & \\
\hline Ex & 63 (30.3) & 73 (35.6) & $86(31.7)$ & & \\
\hline Current & $5(2.4)$ & $5(2.4)$ & $8(3.0)$ & & \\
\hline Patients with severe asthma & $84(40.4)$ & 68 (33.3) & $179(66.1)$ & 0.138 & $<0.001$ \\
\hline
\end{tabular}

Data are presented as mean \pm SD or $n$ (\%), unless otherwise stated. Low: never-on-salmeterol/fluticasone combination (SFC) versus low-dose; high: never-on-SFC versus high-dose; Mini AQLQ: Mini Asthma Quality of Life Questionnaire; ATAQ: Asthma Therapy Assessment Questionnaire; FEV1: forced expiratory volume in one second. \#. patients who were never receiving both inhaled corticosteroids and long-acting $\beta$-agonists; ${ }^{\bullet}$ : derived from an unpaired t-test and Chi-squared test for continuous and categorical variables for low and high, respectively.

the noncombined form of fluticasone $(500 \mu \mathrm{g}$ b.i.d) and salmeterol (50 $\mu \mathrm{g}$ b.i.d) and fluticasone (500 $\mu \mathrm{g}$ b.i.d) alone, AUBIER et al. [5] reported improved peak expiratory flow rates for the high-dose SFC group compared with the fluticasone alone group, but no statistically significant differences between treatment groups with respect to forced expiratory volume in one second (FEV1), daytime or night-time symptoms, and the need for rescue medication. Although the Gaining Optimal Asthma ControL (GOAL) study demonstrated that more uncontrolled patients were able to achieve control with SFC compared with fluticasone propionate alone, GOAL also demonstrated that most patients who achieved control did so at the lowest dose of combination treatment used for the stratum [6].

However, current SFC prescribing patterns, even in the absence of established clinical evidence, show that care providers prescribe high-dose SFC to many difficult-to-treat patients or to those who have failed to respond to low-dose SFC. It is also well established that pulmonary function outcomes on which the SFC efficacy claim is primarily based do not fully reflect a patient's level of asthma control [1, 2, 7-10]. Thus, research is merited to investigate the effects of low- and high-dose SFC on the many dimensions of a patient's asthma control. The present study assessed the response to low-dose SFC and high-dose SFC in severe or difficult-to-treat asthma by longitudinally comparing patients who were on either low- or high-dose SFC to those who were on long-term asthma medications such as ICS or LABA, but not on both, using health outcomes consistent with components of asthma control.

\section{MATERIALS AND METHODS}

\section{Study overview}

The Epidemiology and Natural History of Asthma: Outcomes and Treatment Regimens (TENOR) was a 3-yr multicentre, prospective cohort study of 4,756 subjects aged $\geqslant 6$ yrs with severe or difficult-to-treat asthma enrolled between January and October 2001 [11]. As TENOR was an observational study, there was no experimental intervention. Subjects continued to receive medication and treatment administered for their asthma as indicated by their physician. Patients had to be receiving care from their physician/provider for $\geqslant 1 \mathrm{yr}$ and had to have evidence of either high use of the healthcare system or medications,or both. Patients were excluded if they were heavy smokers ( $\geqslant 30$ pack-yrs) or if they had a diagnosis of cystic fibrosis. The TENOR protocol was approved by a central institutional review board (Independent Reviewing Consultancy, Inc., San Anselmo, CA, USA) and by each centre's institutional review board, where necessary.

\section{Study population and definition of treatment groups}

Patients were included if they were aged $\geqslant 18$ yrs and had complete data on measures of the study outcomes at baseline and at 24-month follow-up. In addition, patients who could be categorised into one of the three treatment groups based on medication use at baseline, 12- and 24-month follow-up visits were included. The two SFC groups, low-dose SFC (100/50 or $250 / 50 \mu \mathrm{g}, \mathrm{n}=205)$ and high-dose SFC (500/50 $\mu \mathrm{g}, \mathrm{n}=271)$, were limited to patients who initiated SFC therapy between the baseline and 12-month visit and remained on this dose of therapy at the 12- and 24-month follow-up visit. For all three visit assessments, the never-on-SFC group was allowed to use an ICS or a LABA, but were never on both ICS and LABA $(n=208)$.

Of 3,489 patients aged $\geqslant 18$ yrs in TENOR, 1,448 were excluded because they did not have treatment assessments at the baseline, 12- and 24-month visits; an additional 1,360 patients were excluded because they did not fit into one of the three treatment cohorts. For specific outcomes, up to 306 
additional patients were excluded due to missing data. The demographic and baseline characteristics of excluded TENOR subjects were similar to those of the TENOR subjects included in the present study.

\section{Study outcomes}

The study outcomes were assessed at baseline and the 24month follow-up visit, which included asthma-related quality of life (ARQoL), asthma control problems, lung function, asthma exacerbations and physician-assessed asthma severity. The ARQoL was measured using the validated Mini Asthma Quality of Life Questionnaire (MiniAQLQ) [12]. The MiniAQLQ measures functional impairments in four different domains that are most troublesome to subjects with asthma. The overall summary scores were analysed, which ranged 1-7, with higher scores indicating better ARQoL. Asthma control problems were assessed using the validated Asthma Therapy Assessment Questionnaire (ATAQ) [13, 14]. The ATAQ includes questions about the following control problems: nocturnal wakening, interference with activities, overuse of reliever medications and self-perception of poor control. ATAQ scores range from zero control problems to four control problems and reflect asthma control in the past 4 weeks to 12 months. Lung function was measured using pre-bronchodilator FEV1 \% predicted. An asthma exacerbation was defined as at least one of any of the following in the previous 3 months: hospitalisation for asthma, emergency room visit for asthma, steroid burst or current use of systemic corticosteroids. Asthma severity was determined subjectively by TENOR investigators as mild, moderate or severe at each visit; the proportion of patients who were categorised as severe between treatment groups were compared.

\section{Statistical analysis}

The demographic and clinical characteristics between treatment groups at baseline were compared using an unpaired t-test for continuous variables and Chi-squared tests for categorical variables. ARQoL, asthma control problems and FEV1 were considered to be continuous, while asthma exacerbations and severity were considered to be categorical (dichotomous).

Linear regression was used to estimate least squares mean differences for continuous outcomes and logistic regression to estimate odds ratios for categorical outcomes at the 24-month follow-up visit, to compare the low-dose SFC group versus the never-on-SFC group, and the high-dose SFC group versus the never-on-SFC group. Unadjusted models only included the independent indicator variable for treatment group (low-dose versus never-on-SFC and high-dose versus never-on-SFC) to estimate the parameter of interest, its corresponding SE, and test statistic.

Since differences in baseline characteristics between treatment groups were expected, propensity scores [15] were used to adjust for treatment selection bias and other potential confounding variables in the comparison of study outcomes between treatment groups. A stepwise logistic regression algorithm ( $p<0.10$ to enter, and $p<0.05$ to remain) was applied to determine which covariates to include in the final models that predicted the propensity to be in a particular treatment group. Variables included in the final propensity score models were age, aspirin sensitivity, indication of moisture present in the home, diabetes status, family history of allergic rhinitis, baseline measures of physician-assessed severity, MiniAQLQ, exacerbation and FEV1. The ability of the propensity score models to discriminate between the treatment cohorts was evaluated using the area under the receiver operating characteristic (ROC) curve, and the model fit was assessed using the Hosmer-Lemeshow Chi-squared test. Patients were categorised into propensity-matched quintiles based on the results of the logistic regression models. The adjusted regression models included the treatment group indicator, the propensity-matched quintiles and the outcome values measured at baseline. The present authors did not adjust for a physician cluster effect due to the limited number of treated patients per physician.

\section{RESULTS}

Of the 208 patients in the control group at baseline, $177(85.1 \%)$ were receiving ICS while $10(4.8 \%)$ were receiving LABA. Of the control group patients neither on ICS nor LABA medications, seven $(3.4 \%)$ were on other long-term asthma controllers, including leukotriene modifiers, while the remaining $14(6.7 \%)$ were not on any long-term controller medications at baseline. Baseline demographic and clinical characteristics, including measures of the study outcomes at baseline for the three treatment groups, are displayed in table 1. Smoking status, age, and sex were similar between treatment groups. The high-dose SFC group had a younger age at asthma onset and a higher proportion of minorities compared with the never-on-SFC group, conditions predictive of fixed airway obstruction [16]. Immunoglobulin E levels, skin test results, history of allergic rhinitis or atopic dermatitis, education, employment status or type of healthcare insurance did not significantly differ between treatment groups (data not shown; $p$-value $>0.05$ for all comparisons). Statistically significant differences in many of the outcome measures at baseline were observed between the treatment groups. Summary statistics for

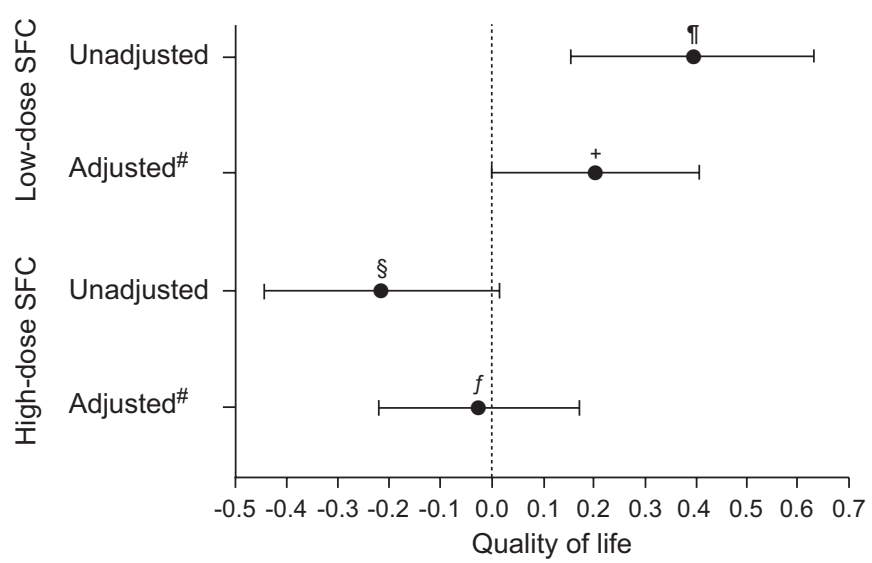

FIGURE 1. Least mean square difference and $95 \%$ confidence intervals (lowdose salmeterol/fluticasone combination (SFC) minus never-on-SFC; high-dose SFC minus never-on-SFC) in mean Mini Asthma Quality of Life Questionnaire overal scores at 24 months. ${ }^{*}$ : baseline differences between treatment groups, including severity differences, were adjusted with propensity scores. ": quality of life (QoL) $0.40, p=0.0015 ;{ }^{+}:$QoL 0.20, p=0.0456; ${ }^{\text {s: }}$ QoL 0.23, p=0.0672; f: QoL 0.01, $\mathrm{p}=0.8915$ 


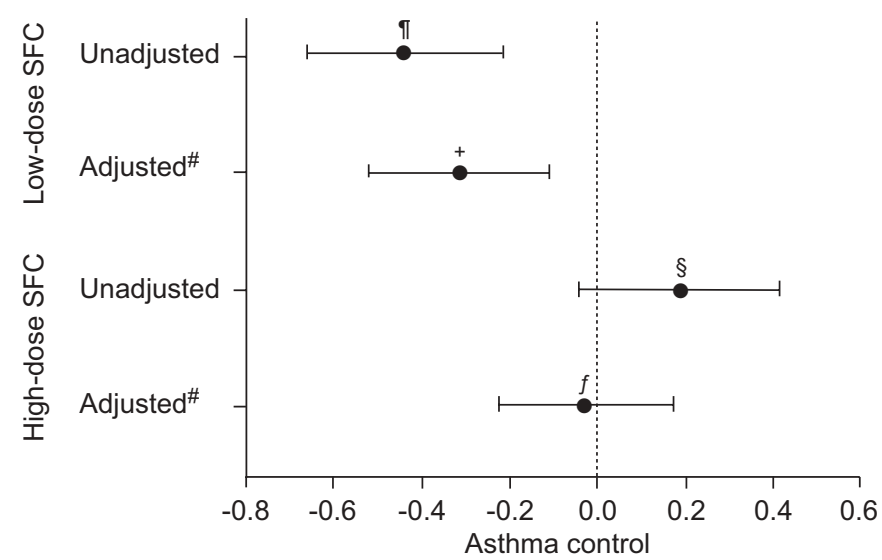

FIGURE 2. Least mean square difference and $95 \%$ confidence intervals (lowdose salmeterol/fluticasone combination (SFC) minus never-on-SFC; high-dose SFC minus never-on-SFC) in mean Asthma Therapy Assessment Questionnaire scores at 24 months. \#: baseline differences between treatment groups, including severity differences, were adjusted with propensity scores. ${ }^{\circ}$ : asthma control -0.46 , $p<0.0001 ;^{+}$: asthma control $-0.33, p=0.0018 ;^{\text {s }}$ : asthma control $0.18, p=0.1328$; $^{f}$ : asthma control $-0.04, p=0.7428$.

outcome measures at baseline showed that the high-dose SFC group had more poorly controlled asthma (i.e. lower MiniAQLQ scores, lower FEV1 scores, higher ATAQ scores, higher proportion of patients with an asthma exacerbation, and a higher proportion considered to have severe asthma) than the never-on-SFC group; the never-on-SFC group had more poorly controlled asthma than the low-dose SFC group.

The propensity score models showed fair discrimination between the high-dose SFC and never-on-SFC groups (area under the ROC curve 0.68) and between the low-dose SFC and never-on-SFC groups (area under the ROC curve=0.66). Hosmer-Lemeshow Chi-squared tests indicated that both models were well calibrated (lack of fit: $\mathrm{p}=0.30$ for low-dose SFC versus never-on-SFC, $\mathrm{p}=0.40$ for high-dose SFC versus never-on-SFC). Baseline characteristics were similar between treatment groups after stratifying by quintile.

Figures 1-5 display the unadjusted and adjusted differences in the 24-month outcomes between low-dose/high-dose SFC and the never-on-SFC group. Compared with the never-on-SFC group, unadjusted mean differences in the overall MiniAQLQ scores were 0.40 (95\% confidence interval (CI) 0.15-0.64) for the low-dose SFC group and - 0.23 (95\% CI -0.47-0.02) for the highdose SFC group (fig. 1). The adjusted mean difference in the overall MiniAQLQ scores was 0.20 (95\% CI 0.00-0.40) for the low-dose SFC group compared with the never-on-SFC group, but the difference between the high-dose SFC group and the never-on-SFC group was -0.01 (95\% CI -0.21-0.18).

After adjustment, the low-dose SFC group compared with the never-on-SFC group had an ATAQ score mean difference of $-0.33(95 \%$ CI $-0.54--0.12)$, whereas the adjusted mean difference for the high-dose SFC group compared with the never-on-SFC group was -0.04 (95\% CI -0.24-0.17; fig. 2). Mean difference in adjusted FEV1 \% pred pre-bronchodilator values was $1.93 \%$ (95\% CI $-0.93-4.80 \%)$ for the low-dose SFC group versus the never-on-SFC group, and was $2.93 \%$ (95\% CI

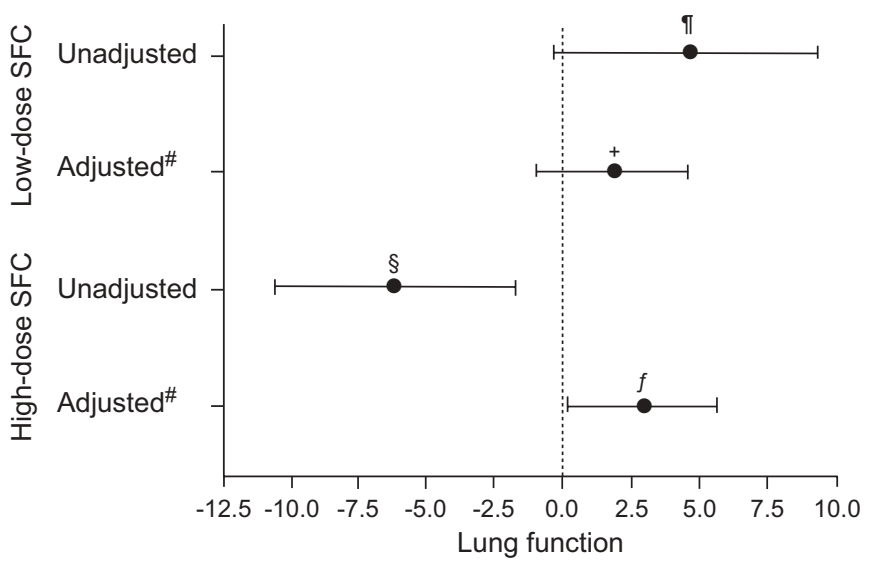

FIGURE 3. Least mean square difference and 95\% confidence intervals (lowdose salmeterol/fluticasone combination (SFC) minus never-on-SFC; high-dose SFC minus never-on-SFC) in mean forced expiratory volume \% predicted prebronchodilator values at 24 months. *: baseline differences between treatment groups, including severity differences, were adjusted with propensity scores. " : lung function 4.61, $p=0.0629$; +: lung function 1.93, $p=0.1866 ;$; : lung function -5.93 , $\mathrm{p}=0.0077 ;$ f: lung function $2.93, \mathrm{p}=0.0462$

0.06-5.81\%) for the high-dose SFC group versus the never-onSFC group (fig. 3).

After adjustment, the odds of having severe asthma was 0.76 (95\% CI 0.43-1.34) for the low-dose SFC group compared with the never-on-SFC group, but was 2.16 (95\% CI 1.35-3.45) for the high-dose SFC group compared with the never-on-SFC group (fig. 4). After adjustment, no statistically significant differences in exacerbations were observed between either the high-dose or low-dose SFC group and the never-on-SFC group $(\mathrm{p}=0.959$ and $\mathrm{p}=0.110$, respectively; fig. 5).

\section{DISCUSSION}

The present analysis examined the response of low- and highdose SFC on health outcomes consistent with components of asthma control in a large cohort of patients with severe or difficult-to-treat asthma. Observed differences in asthmarelated health outcomes at baseline confirmed that low-dose SFC was prescribed to patients who had better asthma control than the never-on-SFC group, and that high-dose SFC was prescribed to patients who had the lowest levels of asthma control. Unadjusted observed differences at the 24-month follow-up visit followed this trend; the low-dose SFC cohort achieved better asthma control than the never-on-SFC group, and the never-on-SFC group achieved better asthma control compared with the high-dose SFC group. The propensity score method was used to adjust for treatment selection bias and other potential confounding.

After adjustment for the propensity to be in a particular treatment cohort and for the baseline outcome values, the lowdose SFC group had significantly more favourable 24-month outcomes than the never-on-SFC group with respect to the differences in MiniAQLQ and ATAQ, and trended toward more favourable FEV1 and asthma severity outcomes. Many of the adjusted outcomes were comparable between the highdose SFC and the never-on-SFC group. These findings suggest that some, if not many, of the high-dose SFC patients showed 


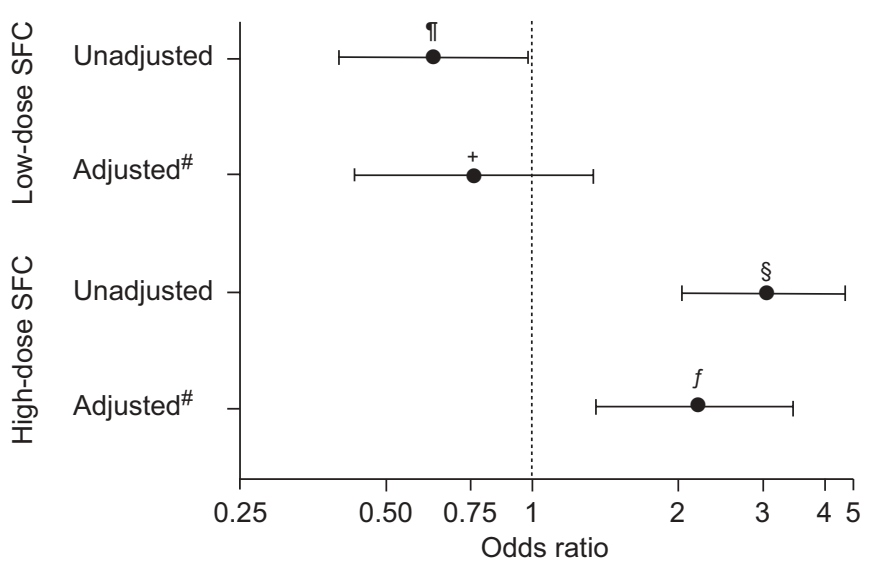

FIGURE 4. Odds ratio (OR) and 95\% confidence intervals (high-dose salmeterol/fluticasone combination (SFC) versus never-on-SFC; low-dose SFC versus never-on-SFC) of severe asthma risk at 24 months. ${ }^{*}$ : baseline differences between treatment groups, including severity differences, were adjusted with propensity scores. ': OR $0.62, p=0.0367$; +: OR $0.76, p=0.3441$; ${ }^{\S}$ : OR 2.99 $\mathrm{p}<0.0001 ;{ }^{f}$ : OR 2.16, $\mathrm{p}=0.0013$.

limited clinical benefit with high-dose SFC. The explanation for the failure of the high-dose SFC cohort to demonstrate substantial improvements in measures of asthma control above and beyond patients not treated with SFC may include inherent, not easily quantifiable, differences between the patients who receive and do not receive high-dose SFC in clinical practice. However, an alternative explanation is that the benefits derived from SFC are optimally achieved with the low-dose SFC and that incremental doses of SFC do not provide added clinical benefit in the severe or difficult-to-treat asthma population.

The current findings suggest a lack of dose-response for SFC. A failure to respond to high-dose SFC in the severe or difficult-totreat population could be explained by the presence of fixed airway resistance, molecular mechanisms of steroid resistance $[17,18]$, a steroid-insensitive form of inflammation [19] or distal lung inflammation not accessible to inhaled medications [20]. The modest high-dose SFC benefit is consistent with the findings of: AUBIER et al. [5], who reported limited benefits of high-dose SFC compared with its alternatives; SzEFLER et al. [21], who found a lack of dose-response for ICS; and MARTIN et al. [22], who demonstrated that ICS nonresponders did not achieve better asthma control when compared with placebo nonresponders. It is possible that more nonresponders were given high-dose SFC compared with low-dose SFC and, therefore, the incremental benefit of high-dose SFC was not observed because of nonresponders. The validity of the observational research will hinge on the findings of similar but independent studies, with or without randomised designs.

The clinical interpretation of the observed differences should be considered carefully. For example, the largest observed mean difference in the total MiniAQLQ scores was 0.40 (in favour of the unadjusted low-dose SFC compared with the never-on-SFC group). Since 0.5 has been found to be the minimal important difference in the AQLQ outcome [23], it could be argued that both the unadjusted and adjusted observed differences in the analogous MiniAQLQ outcome did not achieve clinical significance.

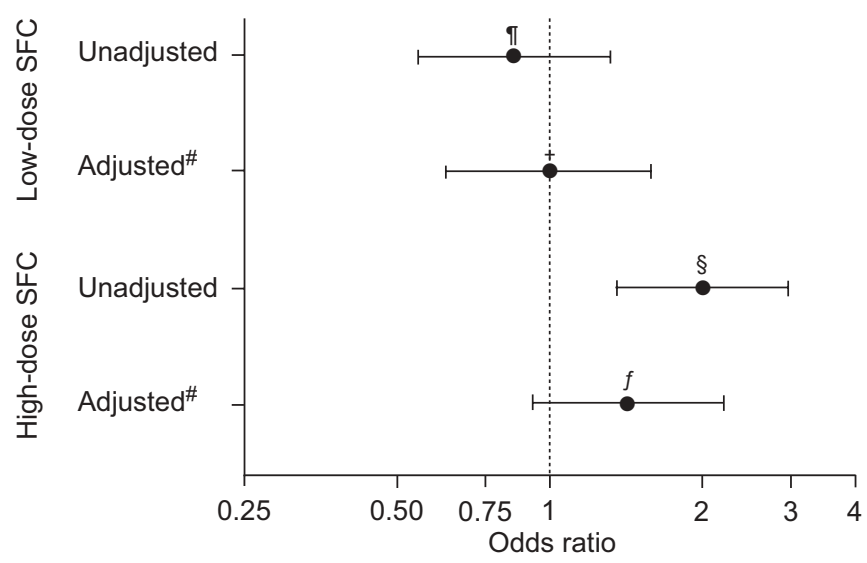

FIGURE 5. Odds ratio (OR) and 95\% confidence intervals (high-dose salmeterol/fluticasone combination (SFC) versus never-on-SFC; low-dose SFC versus never-on-SFC) of the risk of experiencing asthma exacerbations at 24 months. \#: baseline differences between treatment groups, including severity differences, were adjusted with propensity scores. ${ }^{\circ}$ : OR $0.85, p=0.4603 ;{ }^{+}$: OR $0.99, p=0.9592 ;{ }^{\S}$ : OR 1.99, $p=0.0005 ;{ }^{f}$ : OR 1.42, $p=0.1096$.

There are several limitations to the present study that are noteworthy. Medication utilisation was captured in TENOR from an annual study coordinator interview. Therefore, limited data are available to assess or verify compliance or persistence. However, the current study design restricted the SFC groups to those who started SFC treatment between the baseline and 12month follow-up visit, and patients were required to remain on this dose of SFC treatment throughout the 24-month followup visit. The comparison group comprised TENOR adults who were not receiving SFC at any of the visits (baseline, 12- or 24month follow-up) but could have been receiving an ICS or LABA medication. As with any observational study, confounding variables may mask the true effect that SFC has on asthma outcomes.

While confounding may still remain, propensity score methods were applied to adjust for potential confounders that were measured within the TENOR dataset. Arguably, covariate adjustment remains a slightly more common technique for addressing potential confounding, but COOK and GOLDMAN [24] showed that the propensity score methodology is often more robust. The propensity score approach received further endorsement by LAUER [25] who suggested that propensity scores may be the only viable method to answer many questions that arise from clinical practice. Although the present authors evaluated the fit of the propensity score models and the ability of the propensity score models to discriminate between the treatment cohorts, there is no way to test whether other unmeasured variables could have led to a different and better propensity score adjustment. Additionally, the propensity score adjustment depends upon the accurate and precise measurement of the variables that contribute to the model; thus, possible residual confounding in the current analysis cannot be ruled out.

The current results support evidence that some people with asthma achieve better control while taking low-dose salmeterol/ fluticasone combination, but also suggest that high-dose salmeterol/fluticasone combination fails to provide a clinically 
significant benefit in terms of many control-related health outcomes as compared with similar patients not taking salmeterol/fluticasone combination. These findings suggest a limited value of high-dose salmeterol/fluticasone combination. While additional studies are needed, the present findings call for alternative therapeutic approaches in severe/difficult-to-treat asthma for those unable to attain asthma control with or without salmeterol/fluticasone combination.

\section{ACKNOWLEDGEMENTS}

The authors wish to thank D.P. Miller (ICON Clinical Research, San Francisco, CA, USA) for statistical advice, C. Mamay and J. Pena for help with the figures, and J. Zazzali (all Genentech, San Francisco) for constructive critiques of the manuscript.

\section{REFERENCES}

1 National Heart, Lung and Blood Institute, National Institutes of Health. National Asthma Education and Prevention Program. Expert Panel Report 3: Guidelines for the Diagnosis and Management of Asthma 2007. www.nhlibi.nih.gov/guidelines/asthma/asthgdln.htm Date last accessed: May 1, 2008. Date last updated: 2007.

2 Global Initiative for Asthma. Global Strategy for Asthma Management and Prevention 2006. www.ginasthma.com/ Guidelines.asp??11=2\&12=1\&intId =60 Date last accessed: May 1, 2008. Date last updated: 2007.

3 GlaxoSmithKline. Salmeterol/fluticasone propionate combination (Advair) prescribing information. http://us.gsk.com/ products/assets/us_advair.pdf Date last accessed: May 1, 2008. Date last updated: 2007.

4 Nelson HS. Advair: combination treatment with fluticasone propionate/salmeterol in the treatment of asthma. J Allergy Clin Immunol 2001; 107: 398-416.

5 Aubier M, Pieters WR, Schlosser NJ, Steinmetz KO. Salmeterol/fluticasone propionate $(50 / 500 \mathrm{microg})$ in combination in a Diskus inhaler (Seretide) is effective and safe in the treatment of steroid-dependent asthma. Respir Med 1999; 93: 876-884.

6 Bateman ED, Boushey HA, Bousquet J, et al. Can guidelinedefined asthma control be achieved? The Gaining Optimal Asthma ControL study. Am J Respir Crit Care Med 2004; 170: 836-844.

7 Carranza Rosenzweig JR, Edwards L, Lincourt W, Dorinsky P, ZuWallack RL. The relationship between health-related quality of life, lung function and daily symptoms in patients with persistent asthma. Respir Med 2004; 98: 1157-1165.

8 Miller MK, Johnson C, Miller DP, et al. Severity assessment in asthma: an evolving concept. J Allergy Clin Immunol 2005; 116: 990-995.
9 Stoloff SW, Boushey HA. Severity, control, and responsiveness in asthma. J Allergy Clin Immunol 2006; 117: 544-548.

10 Teeter JG, Bleecker ER. Relationship between airway obstruction and respiratory symptoms in adult asthmatics. Chest 1998; 113: 272-277.

11 Dolan CM, Fraher KE, Bleecker ER, et al. Design and baseline characteristics of the epidemiology and natural history of asthma: outcomes and treatment regimens (TENOR) study: a large cohort of patients with severe or difficult-to-treat asthma. Ann Allergy Asthma Immunol 2004; 92: 32-39.

12 Juniper EF, Guyatt GH, Cox FM, Ferrie PJ, King DR. Development and validation of the Mini Asthma Quality of Life Questionnaire. Eur Respir J 1999; 14: 32-38.

13 Vollmer WM, Markson LE, O'Connor E, Frazier EA, Berger M, Buist AS. Association of asthma control with health care utilization: a prospective evaluation. Am J Respir Crit Care Med 2002; 165: 195-199.

14 Vollmer WM, Markson LE, O'Connor E, et al. Association of asthma control with health care utilization and quality of life. Am J Respir Crit Care Med 1999; 160: 1647-1652.

15 Rubin DB. Estimating causal effects from large data sets using propensity scores. Ann Intern Med 1997; 127: 757-763.

16 Bumbacea D, Campbell D, Nguyen L, et al. Parameters associated with persistent airflow obstruction in chronic severe asthma. Eur Respir J 2004; 24: 122-128.

17 Barnes PJ. Corticosteroid resistance in airway disease. Proc Am Thorac Soc 2004; 1: 264-268.

18 Adcock IM. Steroid resistance in asthma. Molecular mechanisms. Am J Respir Crit Care Med 1996; 154: S58-S61.

19 Wenzel SE, Szefler SJ, Leung DY, Sloan SI, Rex MD, Martin RJ. Bronchoscopic evaluation of severe asthma. Persistent inflammation associated with high dose glucocorticoids. Am J Respir Crit Care Med 1997; 156: 737-743.

20 Balzar S, Chu HW, Strand M, Wenzel S. Relationship of small airway chymase-positive mast cells and lung function in severe asthma. Am J Respir Crit Care Med 2005; 171: 431-439.

21 Szefler SJ, Martin RJ, King TS, et al. Significant variability in response to inhaled corticosteroids for persistent asthma. J Allergy Clin Immunol 2002; 109: 410-418.

22 Martin RJ, Szefler SJ, King TS, et al. The Predicting Response to Inhaled Corticosteroid Efficacy (PRICE) trial. J Allergy Clin Immunol 2007; 119: 73-80.

23 Juniper EF, Guyatt GH, Willan A, Griffith LE. Determining a minimal important change in a disease-specific Quality of Life Questionnaire. J Clin Epidemiol 1994; 47: 81-87.

24 Cook EF, Goldman L. Performance of tests based on stratification by a multivariate confounder score or by a propensity score. J Clin Epidemiol 1989; 42: 317-324.

25 Lauer MS. Primary angioplasty - time is of the essence. JAMA 2000; 283: 2988-2989. 